\title{
Th9 cells in immunity and immunopathological diseases
}

\author{
Markus F. Neurath $^{1}$ - Mark H. Kaplan ${ }^{2}$
}

Received: 14 November 2016 / Accepted: 15 November 2016/Published online: 29 November 2016

(C) Springer-Verlag Berlin Heidelberg 2016

Nine is a magic number in mythology. Starting with the nine deities of Heliopolis in ancient Egypt, the strong association of the number nine with the Chinese dragon (a symbol of magic and power) to the nine muses in Greek mythology (including Kalliope, the muse of epic poetry and science), the number nine has always been associated with extraordinary skills. Thus, it comes as no surprise that $\mathrm{T}$ helper cells producing the cytokine interleukin-9 (IL-9) appear to mediate extraordinary functions. In this issue of Seminars in Immunopathology, we discuss the role of Th9 cells, the T helper subset producing IL-9, in immunity and immunopathological disorders. We have contributed and invited reviews from experts in the Th9 field to discuss the development and function of Th9 cells. The articles span topics ranging from the beginning of the field, to the signals and factors that promote development, to the beneficial and disease-related conditions where Th9 cells have been identified. Interestingly, current findings indicate that such Th9 cells are key players in immunology with a broad range of functions ranging from white to dark magic. Needless to say, in the latter context, the hell described in

This article is a contribution to the special issue on Th9 Cells in Immunity and Immunopathological Diseases - Guest Editors: Mark Kaplan and Markus Neurath

Markus F. Neurath

Markus.Neurath@uk-erlangen.de

Mark H. Kaplan

mkaplan2@iu.edu

1 Department of Medicine 1, Kussmaul Campus for Medical Research \& Ludwig Demling Endoscopy Center of Excellence, University of Erlangen-Nürnberg, 91052 Erlangen, Germany

2 Department of Pediatrics and Herman B Wells Center for Pediatric Research, Indiana University School of Medicine, Indianapolis, IN 46202, USA
Dante's Divine Comedy consisted of nine circles. Prepare to be enchanted by the heaven and hell that are Th9 cells.

\section{The identification of IL-9-secreting T cells and the process of differentiation}

Schmitt and Bopp [1] provide insightful perspective on the initial descriptions of IL-9-secreting T cells long before they were called Th9 cells and were initially thought to be a subset of Th2 cells. They describe the characterization and cloning of IL-9 in the late 1980s and early 1990s and discuss the first experiments showing the cytokine signals that promote IL-9 production from T cells, primarily TGF $\beta$, IL-4, and IL-2. The authors close the article by describing the re-discovery of IL9-secreting T cells as a separate subset that is distinguishable from other subsets in work from the Kuchroo and Stockinger groups that launched the current state of the field.

Kaplan [2] explores the differentiation of Th9 cells in more detail. He describes the cytokine signals and transcription factors that are required for induction of IL-9 production in Th9 cells. He details the regulatory elements that have been identified in the $I l 9$ locus and the transcription factors that bind the Il9 promoter and other regulatory elements. The article crosses over into the other reviews by highlighting where signaling or transcription factor-mutant mice have demonstrated the requirement for Th9 cells in various pathological responses. Kaplan also summarizes work that has been done to define Th9 genes other than IL-9 and summarizes where transcription factor and signaling pathway function are conserved in human cells. Finally, he comments on outstanding questions in this area, including whether a lineage defining transcription factor will be identified for the regulation of $I l 9$ as it has been for other cytokines. 
Meylan and Siegel [3] focus on one of the families of factors that has been repeatedly shown to impact the production of IL-9, namely the tumor necrosis factor receptor superfamily (TNFRSF). The authors summarize the three most potent ligand/receptor pairs that stimulate IL-9 production, OX40L/OX40, TL1A/DR3, and GITRL/GITR. They summarize the evidence for each pathway contributing to responses including allergic inflammation and tumor immunity and highlight that despite the relatedness of these receptor pathways, each ligand/receptor pair seems to function through distinct signaling pathways in the induction of IL-9. The authors also provide new data showing the induction of IL-9 by 11 TNFRSF members. Although some of these effects might be Th subset-specific, based on published reports, it further demonstrates that these receptors make separable contributions to cytokine production from the cell.

\section{The benefit of Th9-dependent immune responses}

Three articles in this journal cover the potential beneficial role of IL-9- and Th9-dependent immune responses. LiconaLimón and coworkers [4] address the role of IL-9 and Th9 cells in the context of parasitic infections. The authors summarize the findings on IL-9 in parasitic infections with special reference to helminth infections in developing countries. Specifically, they describe the protective role of IL-9 in the type 2 immune responses against parasitic helminthes and the pathogenic role of IL-9 in some protozoan infections. They highlight the need for additional studies in the field examining the cellular source of IL-9 under in vivo conditions and some of the recently generated reporter mouse strains (see Table 1) might be very helpful to address this question. However, targeting of IL-9 producing cells appears to be a promising target to potentiate or deplete specific immune functions with important regulatory role in parasitic infections.

The review by Rivera Vargas and colleagues [5] covers the role of IL-9 in oncology. Importantly, recent studies have identified a role for IL-9 in driving anti-cancer immune responses, particularly in melanoma and other solid tumors. IL-9 may be produced by cells surrounding the tumor including lymphocytes. Functionally, IL-9 was found to induce innate and adaptive immune responses favoring anti-cancer immunity and tumor elimination. In addition to IL-9, Th9 cells can produce additional cytokines that may play an important role in anti-cancer immunity. For instance, IL-21 may support the generation of adaptive immune responses against cancer. While there is strong evidence to support a role of IL-9 in murine anti-cancer immunity, the functional role of human Th9 cells in cancer remains to be determined. If human Th9 cells are capable of inducing efficient anti-tumor immune responses, one would predict that Th9 cell-based therapies with immunomodulators, cellular therapy, or chemotherapy could promote potent and long lasting antitumor immunity.

Clark and Schlapbach [6] describe the role of IL-9secreting Th9 cells in skin immunology. Importantly, recent studies in human volunteers have shown that Th9 cells predominantly have markers of skin homing and skin residence. This skin tropism of human Th9 cells is remarkable and may indicate that these cells are involved in protection against extracellular pathogens and fungal antigens. Thus, targeting of human Th9 cells may be of particular relevance for skin immunity and inflammation. Consistent with a role of Th9 cells in human disorders, studies in mice have suggested that murine Th9 cells are actively involved in controlling skin inflammation. However, future studies are necessary to fully delineate the role of skin resident Th9 cells in vivo.

\section{Th9 cells in the pathology of type 2 immunity}

In addition to immune responses where Th9 cells are beneficial to the host, clearly the evolutionary advantage for developing Th9 cells, they can also cause significant pathology in a number of systems. As discussed above Licona-Limón and colleagues [4], Th9 cells play a role in type 2 immunity,

Table 1. Il9 reporter mice that can identify IL-9-producing cells in vivo

\begin{tabular}{|c|c|c|c|c|}
\hline Reporter strain & $\begin{array}{l}\text { Type of } \\
\text { reporter }\end{array}$ & $\begin{array}{l}\text { Endogenous } \\
\text { allele }\end{array}$ & Fidelity and detection & Papers reporting usage \\
\hline Il9-Cre & $\begin{array}{l}\text { mCherry-IRES-Cre } \\
\text { knock-in to } I l 9 \text { BAC }\end{array}$ & Intact & $\begin{array}{l}\text { Cherry reporter was not detected but lineage } \\
\text { tracer identified ILC and a subset of Th9 cells } \\
\text { in several airway models }\end{array}$ & $\begin{array}{l}\text { Wilhelm et al. Nat. Immunol. } \\
\text { 2011, 12:1071. }\end{array}$ \\
\hline$I l 9^{\text {Citrine }}$ & $\begin{array}{l}\text { Citrine knock-in to } \\
\text { first } I l 9 \text { exon }\end{array}$ & Disrupted & Detected Th9 cells in IBD model & $\begin{array}{l}\text { Gerlach et al. Nat. Immunol. } \\
\text { 2014, 15:676. }\end{array}$ \\
\hline INFER & $\begin{array}{l}\text { IRES-EGFP knock-in } 3^{\prime} \\
\text { of } I l 9 \text { coding }\end{array}$ & Intact & $\begin{array}{l}\text { Detected ILC and Th9 cells simultaneous with } \\
\text { endogenous IL-9 production in parasite model }\end{array}$ & $\begin{array}{l}\text { Licona-Limón et al. Immunity } \\
\text { 2013, 39:744. }\end{array}$ \\
\hline$I l 9^{9 \mathrm{er}}$ & $\begin{array}{l}\text { EYFP-IRES-Cre knock-in } \\
\text { to first } I l 9 \text { exon }\end{array}$ & Disrupted & Detected EYFP and lineage tracer in T cells and ILC & $\begin{array}{l}\text { Mohapatra et al. Mucosal } \\
\text { Immunol. 2016, 9:275. }\end{array}$ \\
\hline
\end{tabular}


combining functions with Th2 cells in the elimination of extracellular parasites. However, Th9 cells also contribute to allergic inflammation in a number of atopic disease states including atopic dermatitis, discussed above by Clark and Schlapbach [6], asthma reviewed by Koch and colleagues [7], and food allergy that is discussed in the article by Wang and colleagues [8].

Koch, Sopel, and Finotto [7] provide a thorough and extensive review of the evidence for a pathological role of Th9 cells in allergic asthma. They provide a summary of allergic asthma in patients to lay the context for many studies performed in mouse models and also raise important issues with other cells that might be producing IL-9 in these diseases, including innate lymphoid cells (ILCs) and mast cells. They also place in perspective the contribution of Th9 cells in relation to other $\mathrm{T}$ helper cell subsets that clearly are required for allergic inflammation to develop. The authors then provide an extensive description of models where Th9-promoting or Th9-inhibiting cytokines, receptors, signaling molecules, and transcription factors have been shown to be involved in allergic lung inflammation. The authors complete the review by discussing how Th9 cells and IL-9 elicit effects in the allergic lung, describing effects on hematopoietic cells and lung structural cells.

Wang et al. [8] describe the role of IL-9-producing cells in food allergy. The authors present background on the clinical manifestations of food allergy and discuss the immune processes, including the balance between tolerance and sensitization to food allergens that are required for food allergy to develop. The authors note that many cytokines that promote allergic inflammation also increase IL-9 production from ILC and Th9 cells. They outline evidence for the links between IL9 and mast cell expansion in the gut and the presence of allergen-specific IL-9-producing T cells in children with food allergy. Finally, the authors describe recent evidence of IL-9producing mucosal mast cells as a critical cell type distinguishing the ability of mouse strains to develop food allergy. Whether there is a role for Th9 cells in food allergy, and whether IL-9-secreting mast cells contribute to other type 2 immune responses still needs further investigation.

\section{Autoimmunity}

Two review articles in this issue deal with the role of IL-9 and Th9 cells in potentially autoimmune-mediated diseases. The review by Elyaman and Khoury [9] covers the role of IL-9 and Th9 cells in experimental autoimmune encephalomyelitis (EAE), an animal model of multiple sclerosis (MS) in humans. The authors summarize the source and regulation of Th9 cells in vivo, the influence of IL-9 signaling on both peripheral and local resident cells in EAE and the association between IL-9 and MS disease activity in humans. Although the role of Th9 cells in the pathogenesis of MS still needs further investigation, there is strong evidence that IL-9 may play a regulatory role in autoimmune responses in the brain and that IL-9 expression has a reciprocal relationship with pathogenic Th17 cells. However, additional evidence suggests that in vitro differentiated Th9 cells can induce and augment EAE and peripheral neuritis. Thus, additional studies are required, particularly in patients with MS, to fully understand the functional role of Th9 cells in vivo.

Weigmann and Neurath [10] describe the role of IL-9 and Th9 cells in inflammatory bowel diseases (IBD). One of the key forms of IBD in humans, ulcerative colitis, is associated with marked expansion of IL-9-expressing Th9 cells. In ulcerative colitis, IL-9 production correlated with the severity of colitis activity on endoscopy. Moreover, in a murine model of ulcerative colitis induced by oxazolone, citrine reporter mice revealed an expansion of IL-9-producing cells in the colonic mucosa in vivo. Furthermore, IL-9 deficiency and anti-IL-9 antibody therapy protected from colitis activity suggesting that targeting of IL-9 production may be beneficial in certain forms of colitis. Thus, although much needs to be learned about the signals governing Th9 development and persistent activation in vivo, these findings suggested that anti-IL-9 therapy might be a new approach for therapy in patients with ulcerative colitis.

\section{Future questions and challenges in the Th9 cell field}

The reviews summarized above provide comprehensive insight into the development and function of Th9 cells. They also highlight the many unanswered questions in Th9 cell biology. Where are Th9 cells or other IL-9-producing T cells the most relevant cells types in specific responses? Are there beneficial or detrimental functions of Th9 cells that have not yet been described? What is the mechanism of Th9 cell function in various immune responses and pathologies?

Among the challenges to answering these questions is identifying IL-9-producing cells in vivo. Th9 cells have been difficult to detect, and there could be temporal (looking at the wrong time point) or technical reasons for lack of consistent detection. To surmount this challenge, several groups have made $I l 9$ reporter mice that can identify IL-9-producing cells in vivo (Table 1). Each of these has the advantages and limitations of any reporter; the advantages being easy detection, but the limitations being whether the reporter allele is expressed identically to the $I l 9$ gene, and if the reporter is a knock-in, is there monoallelic expression of the locus that would impact detection of the cells and conclusions from the studies? All of the reporters described thus far can detect IL-9 in ILC and T cells, but it is unclear how faithful they are in other cell types. Even with these caveats, they will be 
important tools in the future exploration of IL-9-dependent immunity.

Finally, all of the reviews in this issue have tried to highlight where Th9 cell development and function is conserved in humans and where there is evidence for Th9 cell activity in patient samples. However, there is still much to define in terms of Th9 cell function in humans. In some cases, the evidence for Th9 cell involvement in human disease is as or more compelling than in mouse models. Yet, we are still defining how Th9 cells function in model systems, and testing these functions in patients will be challenging. Future studies will still need to better link Th9 cells to human disease through genome-wide association of Th9-related genes and ultimately mechanistic studies in vivo.

\section{Prologue to the issue}

As you will see in the following review articles, there is much that we have learned in the 20 years since IL-9 secretion from $\mathrm{T}$ cells was first described, and in the almost 10 years since Th9 cells were defined as a distinct T helper cell subset. Still, there is much to learn, and we hope that Kalliope will inspire us to find answers to the outstanding questions in the field that will allow us to better wield the magic of Th9 cells.

\section{References}

1. Schmitt E, Bopp T (2016) Discovery and initial characterization of Th9 cells: the early years. Semin Immunopathol. doi:10.1007/s00281-016-0610-0

2. Kaplan MH (2016) The transcription factor network in Th9 cells. Semin Immunopathol. doi:10.1007/s00281-016-0600-2

3. Meylan F, Siegel RM (2016) TNF superfamily cytokines in the promotion of Th9 differentiation and immunopathology. Semin Immunopathol. doi:10.1007/s00281-016-0612-y

4. Licona-Limon P, Arias-Rojas A, Olguín-Martínez E (2016) IL-9 and Th9 in parasite immunity. Semin Immunopathol. doi:10.1007/s00281-016-0606-9

5. Rivera Vargas T, Humblin E, Végran F et al (2016) TH9 cells in anti-tumor immunity. Semin Immunopathol. doi:10.1007/s00281016-0599-4

6. Clark RA, Schlapbach C (2016) TH9 cells in skin disorders. Semin Immunopathol. doi:10.1007/s00281-016-0607-8

7. Koch S, Sopel N, Finotto S (2016) Th9 and other IL-9producing cells in allergic asthma. Semin Immunopathol. doi:10.1007/s00281-016-0601-1

8. Wang YH, Shik D, Tomar S, Lee J-B, Chen C-Y, Smith A, Sha W (2016) IL-9-producing cells in the development of IgE-mediated food allergy. Semin Immunopathol. doi:10.1007/s00281-0160605-x

9. Elyaman W, Khoury SJ (2016) Th9 cells in the pathogenesis of EAE and multiple sclerosis. Semin Immunopathol. doi:10.1007/s00281-016-0604-y

10. Weigmann B, Neurath MF (2016) Th9 cells in inflammatory bowel diseases. Semin Immunopathol. doi:10.1007/s00281-016-0603-Z 